\title{
The X-ray versus Radio correlation in a sample of INTEGRAL AGN
}

\author{
F. Panessa* \\ Istituto di Astrofisica Spaziale e Fisica Cosmica (IASF-INAF), via del Fosso del Cavaliere 100, \\ 00133 Roma, Italy \\ E-mail: francesca.panessaliaps.inaf.it
}

\section{Bassani}

Istituto di Astrofisica Spaziale e Fisica Cosmica (IASF-INAF), Via P. Gobetti 101, 40129

Bologna, Italy

E-mail: bassani@iasfbo.inaf.it

\section{A. Bazzano}

Istituto di Astrofisica Spaziale e Fisica Cosmica (IASF-INAF), via del Fosso del Cavaliere 100, 00133 Roma, Italy

E-mail: angela.bazzano@iaps.inaf.it

\section{G. Bicknell}

Research School of Astronomy \& Astrophysics, Mt Stromlo Observatory, Cotter Rd., Weston, ACT 2611, Australia

E-mail: geoff@mso.anu.edu.au

\section{P. Castangia}

Osservatorio Astronomico di Cagliari (OAC-INAF), Loc. Poggio dei Pini, Strada 54, 09012

Capoterra (CA), Italy

E-mail: pcastang@oa-cagliari.inaf.it

\section{E. Maiorano}

Istituto di Astrofisica Spaziale e Fisica Cosmica (IASF-INAF), Via P. Gobetti 101, 40129

Bologna, Italy

E-mail: maiorano@iasfbo.inaf.it

\section{A. Malizia}

Istituto di Astrofisica Spaziale e Fisica Cosmica (IASF-INAF), Via P. Gobetti 101, 40129

Bologna, Italy

E-mail: malizia@iasfbo.inaf.it

\section{P. Parma}

Istituto di Radioastronomia (IRA-INAF), Via P. Gobetti 101, 40129 Bologna, Italy

E-mail: parma@ira.inaf.it

\section{A. Tarchi}

Osservatorio Astronomico di Cagliari (OAC-INAF), Loc. Poggio dei Pini, Strada 54, 09012

Capoterra (CA), Italy

E-mail: atarchi@oa-cagliari.inaf.it 


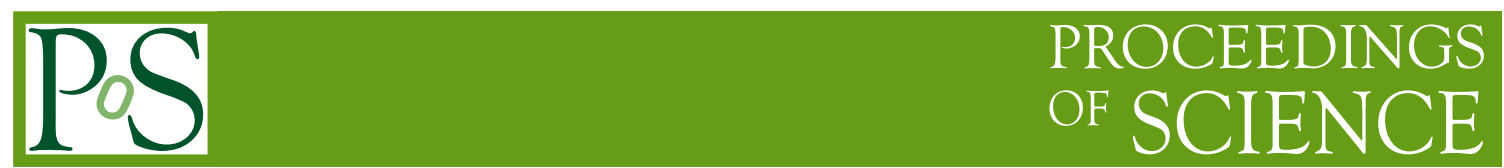

\section{P. Ubertini}

Istituto di Astrofisica Spaziale e Fisica Cosmica (IASF-INAF), via del Fosso del Cavaliere 100, 00133 Roma, Italy

E-mail: pietro.ubertini@iaps.inaf.it

The accretion-ejection mechanism acting in Active Galactic Nuclei (AGN) is one of the main astrophysical open issues, being connected to the role of AGN feedback in galaxy formation evolution studies. The X-ray emission in AGN, associated with the accretion flow, is strongly coupled with the radio emission, associated with a jet. Strong correlations between the X-ray and the radio luminosities are found both in radio-loud (RL) and in radio-quiet (RQ) AGN, despite the fact that in RQ AGN jets are often absent or very weak. For a well defined and complete INTEGRAL RQ AGN sample we have found a strong correlation between the $20-100 \mathrm{keV}$ and the radio luminosity. Black hole mass estimates for half of the sample sources allow us to compute the "fundamental plane of black hole activity", sampling the high luminosity/high accretion rate parameter space.

"An INTEGRAL view of the high-energy sky (the first 10 years)" 9th INTEGRAL Workshop and celebration of the 10th anniversary of the launch,

October 15-19, 2012

Bibliotheque Nationale de France, Paris, France

\footnotetext{
* Speaker.
} 


\section{Introduction}

Active galactic nuclei (AGN) emit continuum radiation from the radio to the hard X-rays, and sometimes gamma-rays. The X-ray and hard X-ray emission in AGN is thought to arise from a non-thermal mechanism such as the Comptonization of the optical-UV disc continuum by hot thermal or non-thermal electrons residing in a magnetically confined gas above the accretion disc (e.g., Haardt \& Maraschi 1991). The radio emission has been classically associated with fast jets carrying relativistic electrons, emitting non-thermal synchrotron radiation. However, the fraction of AGN with powerful relativistic jets is only 10-20\% of the entire AGN population (Kellermann et al. 1989), the majority of which is instead made up of Radio-Quiet (RQ) AGN, defined as having a radio loundness parameter $\mathrm{R} \equiv \mathrm{L}_{6 c m} / \mathrm{L}_{B} \leq 10$. Typically RQ AGN show values of $\mathrm{R}$ concentrated between 0.1-1, while in radio-loud sources the $\mathrm{R}$ values range from 10 to 100 (Kellermann et al. 1989). Lately, Terashima \& Wilson (2003) have introduced the $X$-ray radio-loudness parameter $R_{X}$ $\equiv \mathrm{L}_{v}(6 \mathrm{~cm}) / \mathrm{L}(2-10 \mathrm{keV})$, as the use of the X-ray luminosity with respect to the optical one allows to avoid extinction problems which normally occur in the optical band and which could cause an overestimation of R.

The origin of the radio emission in RQ remains still unclear; it could be ascribed, for instance, to a low-power jet (e.g., Miller, Rawlings \& Saunders 1993), to free-free emission from a molecular torus or to the X-ray corona itself (Gallimore et al. 2004). A radiatively inefficient accretion flow accompanied by a relativistic jet has been invoked to explain both the X-ray and radio emission in low luminosity AGN (LLAGN) (e.g., Narayan \& Yi 1994). This idea has also been supported by the discovery of a "fundamental plane" relation between the X-ray and radio luminosities, and the black hole mass (Merloni et al. 2003; Falcke et al. 2004). A correlation between the X-ray and radio luminosity has been found in Panessa et al. (2007) for a sample of local RQ and RL LLAGN, suggesting that either the source of the X-ray and radio emission is the same or that the two emitting components are physically connected. Here we test the validity of such correlations in a complete sample of relatively high luminosity AGN, as are those detected by the INTEGRAL satellite (e.g., Bird et al. 2010).

\section{The sample}

The sample is extracted from the third INTEGRAL/IBIS survey which lists around 150 (identified and candidates) AGN (Bird et al. 2007). To this large sample, we have applied the Ve/Va relationship to obtain a complete sample of 88 AGN selected in the hard (20-40 keV) X-ray band above $\sim 5$ sigma confidence level (see Malizia et al. 2009 for the detailed definition of the sample); the sample includes 46 Seyfert 1 (including 5 Narrow Line objects), 33 Seyfert 2 and 9 blazars. The 20-100 keV and 2-10 keV luminosities used in this work are taken from Malizia et al. (2009). The 1.4 GHz luminosities have been extracted from the NVSS survey (Condon et al. 2008) directly measured from the maps by using the AIPS software. The NVSS images have 45-arcsec full width at half-maximum angular resolution and nearly uniform sensitivity, with a flux density limit of about $2.5 \mathrm{mJy}$ (Maiorano et al. 2011). Black hole masses have been estimated through different methods (e.g., see Molina et al. 2008). 


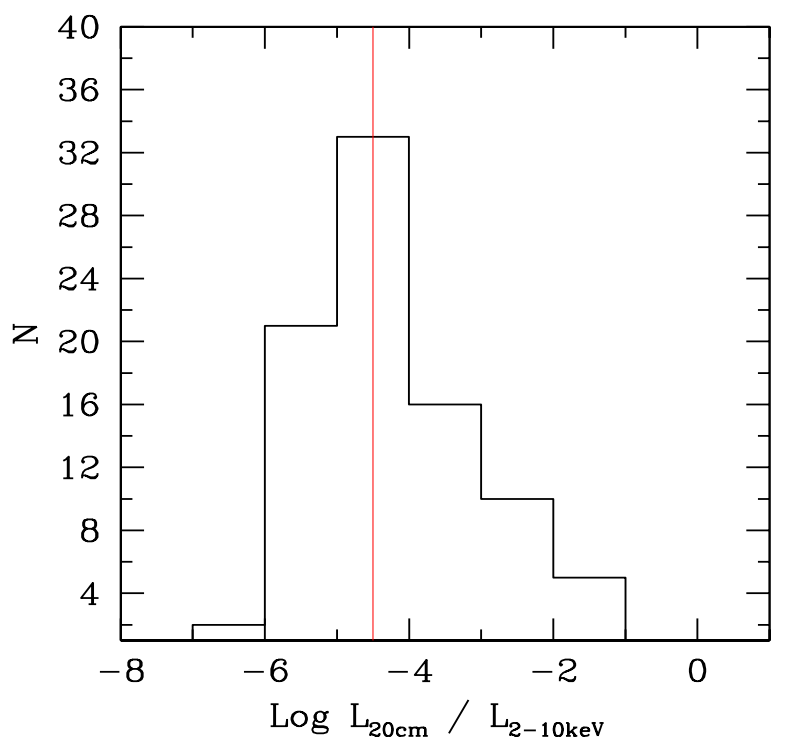

Figure 1: The 2-10 keV versus the $1.4 \mathrm{GHz}$ radio loudness parameter for the INTEGRAL complete sample. The red line is the radio-quiet versus radio loud boundary as in Terashima \& Wilson (2003).

\section{The X-ray radio loudness parameter in luminous AGN}

The X-ray radio loudness distribution of a high energy selected sample is not bimodal as observed in optically selected sample (Kellermann et al. 1989). Terashima \& Wilson (2003) have derived the boundary between the radio-loud and radio-quiet objects to be $\log \mathrm{R}_{X}=-4.5$ (red line in Figure 2 and 3). According to the Terashima \& Wilson (2003) limit, more than half of the INTEGRAL sample sources should be considered as radio-loud AGN. In Panessa et al. (2007) we have redefined this boundary for LLAGN to be $\log \mathrm{R}_{X}=-2.755 \pm 0.015$ (blue line), according to which the majority of the sample are radio quiet AGN. According to these criteria and to the average radio power $\left(\log \mathrm{P}_{20 \mathrm{~cm}} \sim 22-23 \mathrm{~W} \mathrm{~Hz}^{-1} \mathrm{sr}^{-1}\right)$, the sample is mostly made of radio bright AGN but not radio-loud. Indeed, of the 35 type 1 Seyfert galaxies of the sample, only six of them are broad line radio galaxies (see Molina et al. 2007, 2008).

\section{The X-ray versus radio correlation}

A strong correlation has been found between the $1.4 \mathrm{GHz}$ and the $20-100 \mathrm{keV}$ luminosities for the INTEGRAL complete sample (Figure 3). We found two different best-fit linear regression lines for RQ (slope $\alpha \propto 1.0$ ) and RL (slope $\alpha \propto 1.3$ ) AGN. Finally we show the INTEGRAL AGN data on the fundamental plane plot in Figure 4 (blue symbols), compared to the an optically selected sample of nearby Seyfert galaxies (Panessa et al. 2007) (empty symbols). The deviation from the fundamental plane relation (blue line) of the INTEGRAL AGN is mainly due to the RL population, which presents an excess of radio emission.

The presence of a strong correlation between the NVSS and 20-100 keV emission in a sample of AGN selected at hard X-rays supports the idea that, even for efficiently accreting AGN, the 


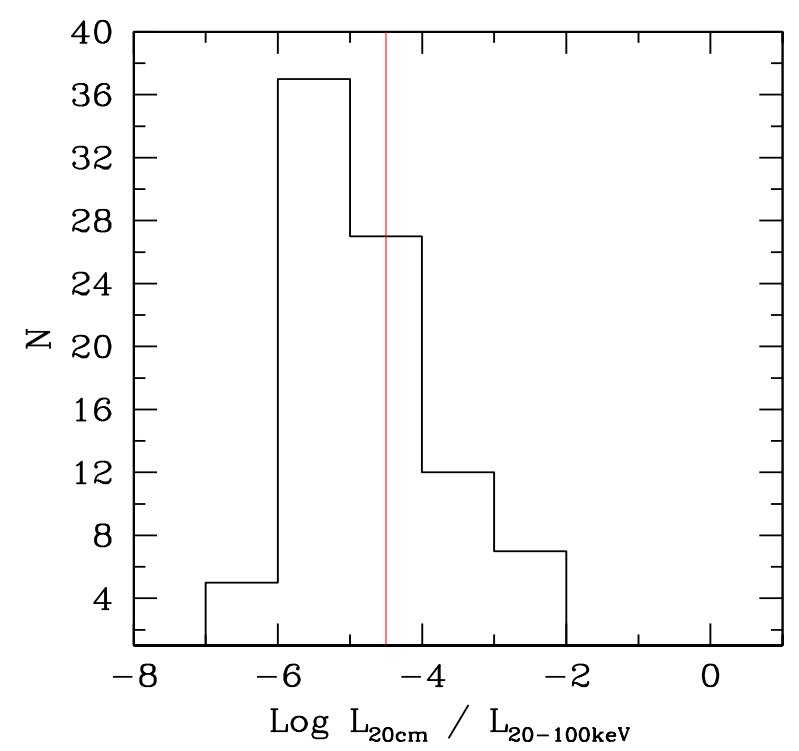

Figure 2: The 20-100 keV versus the $1.4 \mathrm{GHz}$ radio loudness parameter for the INTEGRAL complete sample.

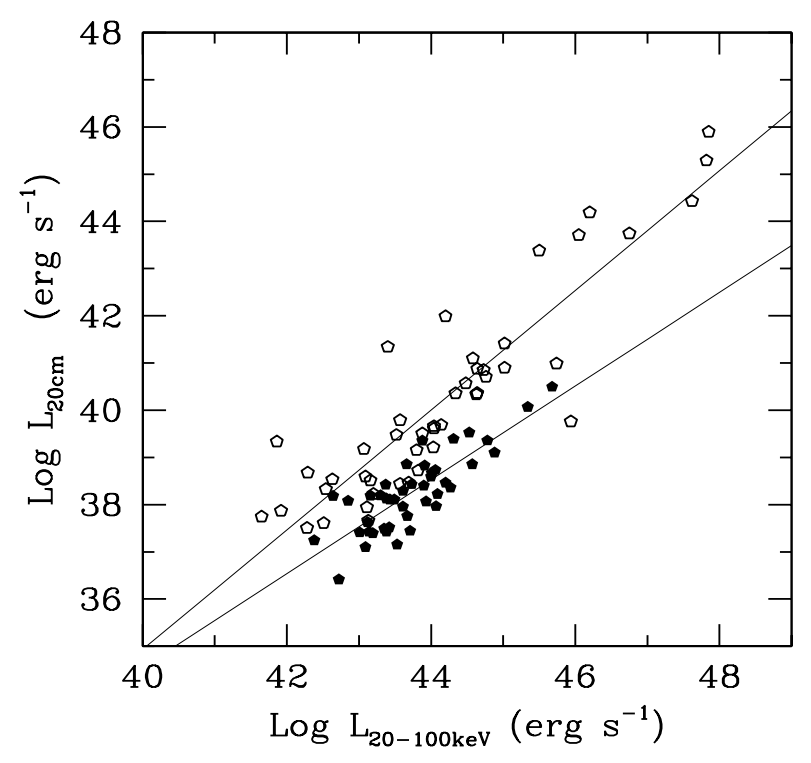

Figure 3: The 1.4 GHz NVSS luminosity versus the 20-100 keV luminosity for the INTEGRAL complete sample together with the best-fit linear regression lines for RQ and RL AGN. 


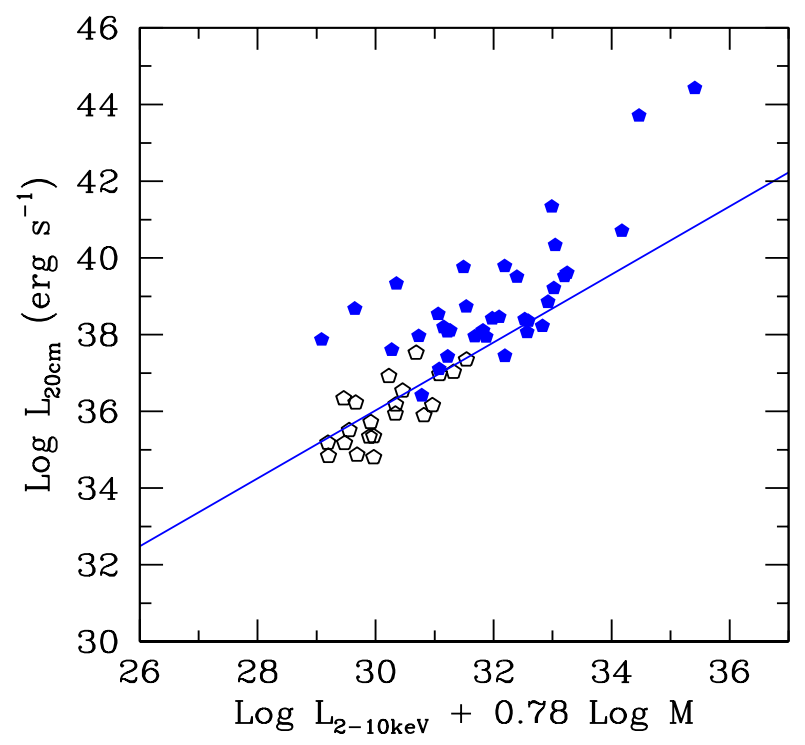

Figure 4: The fundamental plane for black hole activity for the INTEGRAL complete sample (blue symbols) and for the optical selected Seyfert sample (black empty symbols). The blue line represent the fundamental plane equation by Merloni et al. 2003

two physical components are likely connected. However, the fluxes derived using the NVSS data should be taken with caution since they are the total radio flux of the source and may not be representative of the true nuclear radio fluxes. Indeed, at higher spatial resolutions, a large fraction of the radio flux is seemingly resolved (e.g., Giroletti \& Panessa 2009, Orienti et al. 2010), leading to the disappearance of the radio and X-ray correlation (Panessa \& Giroletti, 2013, MNRAS, submitted).

\section{Acknowledgements}

F.P. acknowledges support by INTEGRAL ASI I/033/10/0 and ASI/INAF I/009/10/0.

\section{References}

[1] Anderson, J. M., \& Ulvestad, J. S. 2005, ApJ, 627, 674

[2] Bird, A. J., et al. 2010, ApJS, 186, 1

[3] Cappi, M., et al. 2006, A\&A, 446, 459

[4] Gallimore, J. F., Baum, S. A., \& O’Dea, C. P. 2004, ApJ, 613, 794

[5] Giroletti, M., \& Panessa, F. 2009, ApJL, 706, L260

[6] Haardt, F., \& Maraschi, L. 1991, ApJL, 380, L51

[7] Ho, L. C., Filippenko, A. V., \& Sargent, W. L. W. 1997a, ApJS, 112, 315

[8] Ho, L. C., Filippenko, A. V., \& Sargent, W. L. W. 1997b, ApJ, 487, 568

[9] Ho, L. C. \& Ulvestad, J. S. 2001, ApJS, 133, 77 
[10] Kellermann K. I., Sramek R., Schmidt M., Shaffer D. B., Green R., 1989, AJ, 98,

[11] Maiorano, E., Landi, R., Stephen, J. B., et al. 2011, MNRAS, 416, 531

[12] Malizia, A., Stephen, J. B., Bassani, L., et al. 2009, MNRAS, 399, 944

[13] Merloni, A., Heinz, S., \& di Matteo, T. 2003, MNRAS, 345, 1057

[14] Miller, P., Rawlings, S., \& Saunders, R. 1993, MNRAS, 263, 425

[15] Molina, M., Bassani, L., Malizia, A., et al. 2008, MNRAS, 390, 1217

[16] Molina, M., Giroletti, M., Malizia, A., et al. 2007, MNRAS, 382, 937

[17] Nagar, N. M., Falcke, H., Wilson, A. S., \& Ulvestad, J. S. 2002, A\&A, 392, 53

[18] Narayan, R., \& Yi, I. 1994, ,ApJL, 428, L13

[19] Orienti, M., \& Prieto, M. A. 2010, MNRAS, 401, 2599

[20] Panessa, F., Barcons, X., Bassani, L., et al. 2007, A\&A, 467, 519

[21] Panessa, F., Bassani, L., Cappi, M., et al. 2006, A\&A, 455, 173

[22] Wrobel, J. M., \& Ho, L. C. 2006, ApJL, 646, L95

[23] Terashima, Y. \& Wilson, A. S. 2003, ApJ, 583, 145 